\title{
Piezoelectric mechanism of orientation of a bilayer Wigner crystal in a GaAs matrix
}

\author{
D.V.Fil \\ Institute for Single Crystals National Academy of Sciences of Ukraine, Lenin av. 60 Kharkov 61001 Ukraine \\ e-mail: fil@isc.kharkov.com
}

\begin{abstract}
A mechanism for orientation of bilayer classical Wigner crystals in a piezoelectric medium is considered. For the GaAs system the piezoelectric correction to the electrostatic interaction between electrons is calculated. It is shown that taking into account the correction due to the piezoelectric effect leads to a dependence of the total energy of the electron crystal on its orientation with respect to the crystallographic axes of the surrounding matrix. A generalization of Ewald's method is obtained for calculating the anisotropic interaction between electrons in a Wigner crystal. The method is used to calculate the energy of bilayer Wigner crystals in electron layers parallel to the crystallographic planes (001), (0-11), and (111) as a function of their orientation and the distance between layers, and the energetically most favorable orientation for all types of electron lattices in a bilayer system is found. It is shown that phase transitions between structures with different lattice symmetry in a Wigner crystal can be accompanied by a change of its orientation.
\end{abstract}

\section{INTRODUCTION}

It is known that a system of electrons in the presence of a neutralizing positive background at sufficiently low temperatures and densities goes into a Wigner crystal state. In particular, this situation takes place in two-dimensional electron layers in AlGaAs-GaAs heterojunctions. The formation of a Wigner crystal phase occurs under the condition that the average distance between electrons is much greater than the effective Bohr radius (in the absence of an external magnetic field) or the cyclotron radius (in high magnetic fields). The latter situation corresponds to a filling factor $\nu \ll 1$. In quantum Hall systems the formation of modulated electron structures can occur in other regimes as well. For example, skyrmion lattices can arise in the quantum Hall ferromagnet regime $(\nu \approx 1,1 / 3)$. [1] In low magnetic fields $(\nu \approx N+1 / 2$, where $N$ is an integer and $N \geq 4)$ the formation of stripe structures can occur at the top, partially filled Landau level. The formation of such structures was predicted theoretically [2] and confirmed experimentally [3] by observation of a strong anisotropy of the conductivity.

An interesting question is that of the orientation of the nonuniform electron structures relative to the crystallographic axes of the surrounding matrix. In the two-dimensional electron layers realized in AlGaAs heterostructures, an important influence on the orientation of the electron lattice can be exerted by the piezoelectric interaction between the electron and elastic subsystems. This possibility was first pointed out in relation to Wigner crystals in Refs. [4] and [5]. A piezoelectric mechanism for the orientation of the stripe structure was considered in Ref. [6]. In particular, in that paper an effect was detected wherein a reorientation of the stripes in bilayer systems arises in the case when the period of the structure is greater than the distance between layers. In Ref. [6] the electron subsystem was described using a charge-density-wave model. The purpose of the present paper is to examine the piezoelectric mechanism of orientation of nonuniform electronic structures in bilayer systems in another limiting case, corresponding not to a charge density wave but to a classical Wigner crystal. As in Ref. [6], we use a model which takes into account the anisotropy of the elastic constants of the crystalline matrix. We develop an approach whereby the energy of a system with an anisotropic interaction between electrons can be calculated exactly; this approach is a generalization of Ewald's method for calculating Coulomb sums. As a particular case we obtain results pertaining to a monolayer system. This topic was discussed previously in Ref. [5], where consideration was limited to the model situation of an isotropic crystal. Such a model does not answer the question of the specific orientation that will be realized in the GaAs system, the elastic properties of which are described by three elastic constants rather than two. Furthermore, the method of rapidly convergent lattice sums was not used in Ref. [5]. For the case of bilayer systems, as far as we know, the question of a piezoelectric mechanism of orientation of a Wigner crystal has not been considered before.

The lattice symmetry of a classical Wigner crystal is determined by the minimum of its Coulomb energy. In a monolayer system the minimum is achieved for a hexagonal lattice [7]. In a bilayer system with the same electron density in the two layers, five types of electron lattice can form. The structure, dynamical properties, and melting criterion of such systems have been studied in detail in Refs. [8 10]. Quantum bilayer Wigner crystals in an external magnetic field were treated in Refs. 11 and 12]. The possibility of formation of bilayer Wigner crystals in superfluid helium films was examined in Refs. 13 and 14.

In a classical bilayer Wigner crystal the transition between different crystalline phases is regulated by the parameters $\eta=d \sqrt{n}$, where $d$ is the distance between layers and $n$ is the electron density in the layer. The case $\eta=0$ corresponds to a monolayer system with a doubled density, and the case $\eta=\infty$ to a system of two noninteracting layers. In both 
cases the minimum energy corresponds to hexagonal lattices, with a period differing by a factor of $\sqrt{2}$. Therefore, for finite $\eta$ there must be transition phases: rectangular, square, and rhombic.

The piezoelectric interaction, generally speaking, can lead to a shift of the boundaries between phases. In GaAs the piezoelectric interaction is rather weak, and so the indicated effect will be small. Nevertheless, since the Coulomb interaction in a system with cubic lattice symmetry is isotropic, the piezoelectric correction to the interaction between electrons can be important for determining the orientation of the electron crystal.

\section{ENERGY OF THE PIEZOELECTRIC INTERACTION BETWEEN ELECTRONS IN A WIGNER CRYSTAL}

Consider an infinite piezoelectric medium. The electrostatic potential $\varphi$ of an electron placed at the origin of the coordinate system is given by the following system of equations:

$$
\begin{array}{r}
\operatorname{div} \mathbf{D}=4 \pi e \delta(\mathbf{r}), \\
\frac{\partial \sigma_{i k}}{\partial x_{k}}=0,
\end{array}
$$

where

$$
D_{i}=-\varepsilon_{i k} \frac{\partial \varphi}{\partial x_{k}}-4 \pi \beta_{i, k l} u_{k l}-
$$

is the electric displacement vector, and

$$
\sigma_{i k}=\lambda_{i k l m} u_{l m}-\beta_{l, i k} \frac{\partial \varphi}{\partial x_{l}}-
$$

is the stress tensor. Here $\varepsilon_{i k}$ is the dielectric tensor, $\lambda_{i k l m}$ is the tensor of elastic constants, $\beta_{l, i k}$ is the tensor of piezoelectric moduli, and $u_{i k}$ is the strain tensor. After a transformation to the Fourier components, the system of equations (1) reduces to an algebraic system, and one can easily write the solution for the electrostatic potential. Let us write it out explicitly for a cubic system, the properties of which are determined by three elastic constants $c_{11}, c_{12}$, and $c_{44}$, one piezoelectric modulus $e_{14}$, and the dielectric constant $\varepsilon$ :

$$
\varphi_{\mathbf{q}}=\frac{4 \pi e}{\varepsilon q^{2}}-\frac{(4 \pi)^{2} e}{\varepsilon q^{2}} \chi \frac{P\left(q_{x}, q_{y}, q_{z}\right)}{q^{8} \rho^{3} s_{1}^{2}(\mathbf{q}) s_{2}^{2}(\mathbf{q}) s_{3}^{2}(\mathbf{q})}+O\left(\chi^{2}\right),
$$

where $\chi=e_{14}^{2} / \varepsilon c_{11}$ is a small parameter in which the expansion is done, $s_{i}(\mathbf{q})$ is the velocity of sound with polarization $i$ in the direction $\mathbf{q}$, and $\rho$ is the density of the medium. The function $P$ is a homogeneous 8 th-degree polynomial of the form

$$
P\left(q_{x}, q_{y}, q_{z}\right)=q^{2}\left(a_{1} q_{x}^{2} q_{y}^{2} q_{z}^{2}+a_{2} \sum_{l \neq k} q_{l}^{4} q_{k}^{2}\right)+a_{3} \sum_{l \neq k} q_{l}^{4} q_{k}^{4}
$$

where $l, k=x, y, z$, and

$$
\begin{array}{r}
a_{1}=c_{11}\left(2 c_{12}^{2}-2 c_{11} c_{12}+c_{44}^{2}-2 c_{11} c_{44}\right), \\
a_{2}=c_{11}^{2} c_{44}, \\
a_{3}=\frac{1}{2} c_{11}\left(c_{11}+c_{12}\right)\left(c_{11}-c_{12}-2 c_{44}\right) .
\end{array}
$$

The $x, y, z$ axes are directed along the fourfold axes of the crystalline matrix. As we see from formula (4), the electrostatic potential contains a correction $\delta \varphi_{\mathbf{q}}$, the presence of which is due to the piezoelectric interaction. In an isotropic crystal, in which the sound velocity is independent of the direction and the coefficient $a_{3}$ in (5) is zero, the correction linear in $\chi$ can be represented as an expansion in a finite number of spherical harmonics:

$$
\delta \varphi_{\mathbf{q}}=-\frac{(4 \pi)^{2} e}{\varepsilon q^{2}} \chi \sum_{n} \sum_{m=-n}^{n} A_{n m} Y_{n m}\left(\Theta_{\mathbf{q}}, \psi_{\mathbf{q}}\right)
$$

where $n$ is even and $n \leq 6$. In Eq. (7) $\psi_{\mathbf{q}}$ and $\Theta_{\mathbf{q}}$ are the polar and azimuthal angles, respectively. The coefficients $A_{n m}$ are expressed in terms of the longitudinal and transverse sound velocities. Expansion (7) in the case of an anisotropic 
crystal will also contain higher harmonics allowed by the symmetry of the system. The coefficients $A_{n m}$ for the anisotropic case can be found numerically. A calculation of these coefficients for the GaAs system $\left(c_{11}=12.3 \times 10^{11}\right.$ $\left.\mathrm{dyn} / \mathrm{cm}^{2}, c_{12}=5.7 \times 10^{11} \mathrm{dyn} / \mathrm{cm}^{2}, c_{44}=6.0 \times 10^{11} \mathrm{dyn} / \mathrm{cm}^{2}\right)$ shows that the main contribution to the sum in $(7)$ is given by the same harmonics $(n \leq 6)$ as in the isotropic system (the coefficients of the higher harmonics are at least an order of magnitude smaller). It should be noted that, because the relations between the expansion coefficients are determined by three rather than two elastic constants, the problem cannot be reduced to isotropic even by limiting consideration to the lowest harmonics.

Using expansion (7), one can easily find the correction to the electrostatic interaction between electrons in a piezoelectric medium. After doing the inverse Fourier transformation we find

$$
\delta V(\mathbf{r})=-\frac{e^{2} \chi}{\varepsilon r} G\left(\Theta_{\mathbf{r}}, \psi_{\mathbf{r}}\right)
$$

where

$$
G\left(\Theta_{\mathbf{r}}, \psi_{\mathbf{r}}\right)=4 \pi \sum_{n m} A_{n m}(-1)^{\frac{n}{2}} \frac{n !}{2^{n}[(n / 2) !]^{2}} Y_{n m}\left(\Theta_{\mathbf{r}}, \psi_{\mathbf{r}}\right) .
$$

The form of the function $G$ calculated for the GaAs crystal is shown in Fig. 1 (the coordinate axes are chosen along the fourfold axes). As we see from Eq. (8), the interaction between electrons contains a contribution corresponding to attraction, the strength of which depends on the direction of $\mathbf{r}$ and decays as $1 / r$. Since this decay is as slow as that of the Coulomb interaction, the convergence of the lattice sums will be slow. Therefore, for correct calculation of the piezoelectric correction to the energy of a Wigner crystal it is better use a method of rapidly convergent sums, modified to take into account the anisotropy of the interaction.

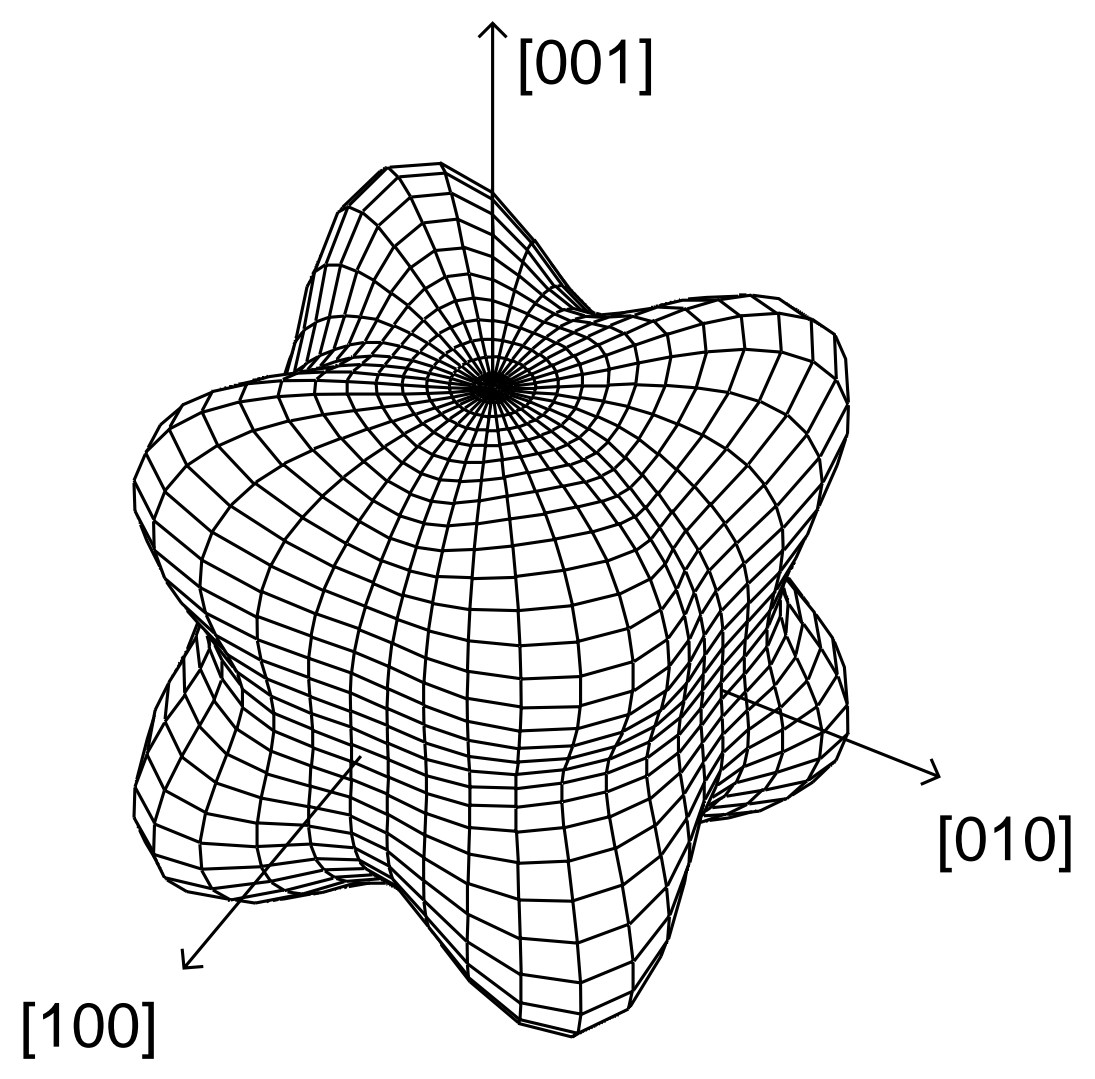

FIG. 1. The anisotropy of piezoelectric interaction between electrons in a GaAs host matrix. 
Consider a bilayer electron system placed in an infinite piezoelectric medium and oriented normal to a certain crystalline direction. In this case it is convenient to make a change of variable in Eq. (9) to the new angles $\Theta$ and $\psi$, measured from the normal to the surface of the electron layer and from an axis lying in the plane of the layer, respectively (the overall structure of expression (9) is preserved - only the values of the expansion coefficients are changed). If the structure of the electron crystal is assumed fixed, then its orientation is determined by the contribution of the terms in (9) which depend on the angle $\psi_{\mathbf{r}}$ in the chosen reference frame. The latter correspond to harmonics with $m \neq 0$. For calculating the lattice sums we rewrite the dependence of the $\psi_{\mathbf{r}}$-dependent part of the interaction between electrons in the form

$$
V_{a n}\left(r, \Theta_{\mathbf{r}}, \psi_{\mathbf{r}}\right)=-\frac{e^{2} \chi}{\varepsilon r} \sum_{l \geq 0} \sum_{|m|>0} B_{l m} \cos ^{l} \Theta_{\mathbf{r}} \sin ^{|m|} \Theta_{\mathbf{r}} e^{i m \psi_{\mathbf{r}}},
$$

Using the explicit form of the spherical harmonics, we can express the coefficients $B_{l m}$ in terms of the coefficients $A_{n m}$. Since the index $n$ in Eqs. (7) and (9) takes on only even values, the coefficients $B_{l m}$ are nonzero only for $l$ and $m$ having the same parity. If we keep a finite number of spherical harmonics in expansion (9), then the sum in (10) will also contain a finite number of terms. We note that to preserve the point symmetry with respect to the angle $\psi$ when a finite number of harmonics is taken into account, the transformation to the indicated coordinate system should be done in Eq. (4) and then the values of the expansion coefficients in (7) should be found numerically.

Taking Eq. (10) into account, we write the anisotropic contribution to the energy of the electron crystal in the form

$$
E_{a n}=E_{a n}^{i n}+E_{a n}^{o u t}+E_{a n}^{B G},
$$

where

$$
E_{a n}^{i n}=-\frac{e^{2} \chi}{\varepsilon} \sum_{|m|>0} B_{0 m} \sum_{\mathbf{R} \neq \mathbf{R}^{\prime}} \frac{1}{\left|\mathbf{R}-\mathbf{R}^{\prime}\right|} e^{i m \psi_{\mathbf{R}^{\prime}-\mathbf{R}}}
$$

corresponds to the contribution of the interaction within the layers,

$$
E_{\text {an }}^{\text {out }}=-\frac{e^{2} \chi}{\varepsilon} \sum_{l \geq 0} \sum_{|m|>0} B_{l m} \sum_{\mathbf{R}, \mathbf{R}^{\prime}} \frac{d^{l}\left|\mathbf{R}-\mathbf{R}^{\prime}-\mathbf{c}\right|^{|m|}}{\left[\left|\mathbf{R}-\mathbf{R}^{\prime}-\mathbf{c}\right|^{2}+d^{2}\right]^{(|m|+l+1) / 2}} e^{i m \psi_{\mathbf{R}^{\prime}+\mathbf{c}-\mathbf{R}}}
$$

describes the contribution of the interlayer interaction, and $E_{\mathrm{an}}^{B G}$ gives the correction to the interaction with the positive neutralizing background. The vectors $\mathbf{R}$ and $\mathbf{R}^{\prime}$ in Eqs. (12) and (13) are lattice vectors, and the vector c specifies the displacement of the upper sublattice relative to the lower. The lattice sums in (12) and (13) can be reduced to a rapidly convergent form with the use of a modified version of Ewald's method (see Appendix). Employing this method gives

$$
E_{a n}=-\frac{N e^{2} \chi}{\varepsilon} \sqrt{n}\left(S_{\text {in }}+S_{\text {out }}\right),
$$

where $N$ is the total number of particles in the layer, and

$$
\begin{gathered}
S_{\text {in }}=\sum_{m} B_{0 m}\left\{\sum_{\mathbf{R} \neq 0} e^{i m \psi_{\mathbf{R}}} \Phi\left(m, \pi n R^{2}\right)+i^{|m|} \sum_{\mathbf{G} \neq 0} e^{i m \psi_{\mathbf{G}}} \Phi\left(m, \frac{G^{2}}{4 \pi n}\right)\right\}, \\
S_{\text {out }}=\sum_{l m} B_{l m}\left\{\sum_{\mathbf{R}} \frac{d^{l}|\mathbf{R}+\mathbf{c}|^{|m|}}{\left[|\mathbf{R}+\mathbf{c}|^{2}+d^{2}\right]^{\frac{|m|+l}{2}}} e^{i m \psi_{\mathbf{R}+\mathbf{c}}} \Phi\left(l+|m|, \pi n\left[|\mathbf{R}+\mathbf{c}|^{2}+d^{2}\right]\right)\right. \\
\left.+i^{|m|} \sum_{\mathbf{G} \neq 0} e^{-i \mathbf{G c}+i m \psi_{\mathbf{G}}} \Psi\left(l, m, \frac{G^{2}}{4 \pi n}, \pi n d^{2}\right)\right\}
\end{gathered}
$$

where $\mathbf{G}$ are reciprocal lattice vectors. In Eqs. (15) and (16) we have introduced the functions

$$
\Phi(m, x)=\sqrt{\frac{\pi}{x}} \frac{\Gamma\left(\frac{|m|+1}{2}, x\right)}{\Gamma\left(\frac{|m|+1}{2}\right)}
$$




$$
\begin{aligned}
& \Psi(l, m, x, y)=\frac{1}{2} \sqrt{\frac{\pi}{x}} \frac{1}{\Gamma\left(\frac{l+|m|+1}{2}\right)} \sum_{s=0}^{N(l, m)} C_{N(l, m)+s}^{2 s}(x y)^{\frac{|m|+l-2 s}{4}} \\
& \times\left[e^{-2 \sqrt{x y}} F(s, \sqrt{x}-\sqrt{y})+(-1)^{\frac{l+|m|-2 s}{2}} e^{2 \sqrt{x y}} F(s, \sqrt{x}+\sqrt{y})\right]
\end{aligned}
$$

In Eq. (18) the $C_{i}^{j}$ are binomial coefficients, $N(l, m)=\max [(|m|-l) / 2,(l-|m|-2) / 2]$, and

$$
F(s, z)=\Gamma(s+1 / 2)-\operatorname{sgn}(z) \gamma\left(s+1 / 2, z^{2}\right) .
$$

In Eqs. (17)-(19) $\Gamma(x)$ is the gamma function, and $\Gamma(k, x)$ and $\gamma(k, x)$ are incomplete gamma functions. We note that for $l, m$ equal to zero (these terms have not been taken into account, since they give a direction-independent correction to the interaction) the sums (15) and (16) reduce to the known expressions for the isotropic case. [7,8]

\section{ORIENTATION OF A BILAYER WIGNER CRYSTAL IN A GaAs MATRIX}

Let us use the results of the previous Section to determine the orientation of bilayer Wigner crystals lying in the planes (001), (0-11), and (111) in the GaAs matrix. In describing the piezoelectric interaction with allowance for anisotropy of the elastic constants, we keep in expansion (7) only the harmonics with $n \leq 18$ and $|m| \leq 12$. We note that in the cases considered below, harmonics with $n>6$ only influence the orientation of hexagonal structures in layers parallel to the (001) plane and that of square structures in layers parallel to the (111) plane. In these cases the symmetry of the system leads to the vanishing of the contribution of the lower harmonics to the energy of the Wigner crystal.

The structure of a Wigner crystal in a bilayer system is specified by the primitive lattice vectors $\mathbf{R}_{1}$ and $\mathbf{R}_{2}$ and the vector $\mathbf{c}$ of the relative displacement of the sublattices in adjacent layers. The values of these vectors for the five types of lattice considered are listed in Table $\mathbb{1}$. The values of $\eta$ for which the change of lattice symmetry occurs were obtained in Ref. 8]. Since we will have need of the functions $\delta(\eta)$ (for a rectangular lattice) and $\alpha(\eta)$ (for a rhombic lattice), we have repeated the calculations of Ref. [8]. According to the results of our calculations, the transition between the rectangular and square phases occurs at $\eta \approx 0.263$, that between the square and rhombic phases at $\eta \approx 0.621$, and that between the rhombic and double hexagonal at $\eta \approx 0.732$. The first two are second-order transitions, and the last is first-order. These results reproduce those of Ref. [8]. (We will not analyze the transition between the simple hexagonal and rectangular phases, which, according to Ref. 88, occurs at $\eta=0.006$, since at this transition the orientation of the electron crystal changes only slightly.)

TABLE I. Structure of double layer Wigner crystals. $\mathbf{R}_{1}, \mathbf{R}_{2}$ are the primitive vectors of the direct lattice; $\mathbf{G}_{1}, \mathbf{G}_{2}$, the

\begin{tabular}{|c|c|c|c|c|c|c|c|}
\hline Lattice type & $\mathbf{R}_{1}$ & $\mathbf{R}_{2}$ & $\mathbf{G}_{1}$ & $\mathrm{G}_{2}$ & c & Variable parameter & $\mathrm{n}$ \\
\hline simple hexagonal & $(a, 0)$ & $(0, \sqrt{3} a)$ & $\left(\frac{2 \pi}{a}, 0\right)$ & $\left(0, \frac{2 \pi}{a \sqrt{3}}\right)$ & $\frac{a}{2}(1, \sqrt{3})$ & - & $\frac{1}{a^{2} \sqrt{3}}$ \\
\hline rectangular & $(a, 0)$ & $(0, a \delta)$ & $\left(\frac{2 \pi}{a}, 0\right)$ & $\left(0, \frac{2 \pi}{a \delta}\right)$ & $\frac{a}{2}(1, \delta)$ & $1<\delta<\sqrt{3}$ & $\frac{1}{a^{2} \delta}$ \\
\hline square & $(a, 0)$ & $(0, a)$ & $\left(\frac{2 \pi}{a}, 0\right)$ & $\left(0, \frac{2 \pi}{a}\right)$ & $\frac{a}{2}(1,1)$ & - & $\frac{1}{a^{2}}$ \\
\hline rhombic & $(a, 0)$ & $a(\cos \alpha, \sin \alpha)$ & $\frac{2 \pi}{a}(1,-\cot \alpha)$ & $\left(0, \frac{2 \pi}{a \sin \alpha}\right)$ & $\frac{a}{2}(1+\cos \alpha, \sin \alpha)$ & $\frac{\pi}{3}<\alpha<\frac{\pi}{2}$ & $\frac{1}{a^{2} \sin \alpha}$ \\
\hline double hexagonal & $(a, 0)$ & $\left(\frac{a}{2}, \frac{a \sqrt{3}}{2}\right)$ & $\frac{2 \pi}{a}\left(1,-\frac{1}{\sqrt{3}}\right)$ & $\left(0, \frac{4 \pi}{a \sqrt{3}}\right)$ & $\frac{a}{2}\left(1, \frac{1}{\sqrt{3}}\right)$ & - & $\frac{2}{a^{2} \sqrt{3}}$ \\
\hline
\end{tabular}
primitive vectors of the reciprocal lattice; $\mathbf{c}$, the vector of relative displacement of sublattices in adjacent layers; $n$, the one layer electron density. 
The anisotropic contribution to the energy of a bilayer electron crystal as a function of its orientation in the (001), $(0-11)$, and (111) planes is shown in Figs. 2, 3, and 4 for various values of the parameter $\eta$ (the energy is given as the energy per electron in units of $\left.e^{2} \chi \sqrt{n} / 2 \varepsilon\right)$.

For a two-dimensional Wigner crystal lying in the (001) plane, the anisotropic contribution to the energy of the simple hexagonal structure does not exceed $2 \times 10^{-2}$ (in the indicated units). A minimum of the energy is reached at an angle between $\mathbf{R}_{1}$ and the [100] axis which is a multiple of $30^{\circ}$. The absolute minimum of the energy of the rectangular phase occurs when one of the primitive lattice vectors is directed along the [100] axis. At small $\eta$ there are also local minima corresponding to angles $\beta \approx \pm 30^{\circ}$ between $\mathbf{R}_{1}$ and one of the fourfold axes. With increasing $\eta$ the local minima vanish, and the anisotropy energy increases substantially. A minimum of the energy of the square phase corresponds to an orientation of the elementary lattice vectors along the fourfold axes. The vector c here is oriented along one of the twofold axes. This orientation of the vector $\mathbf{c}$ is preserved in the rhombic phase as well (the vectors $\mathbf{R}_{1}$ and $\mathbf{R}_{2}$ rotate smoothly on changes in $\eta$ ). The transition to the hexagonal phase as the parameter $\eta$ is increased further is accompanied by a jumplike change in the orientation of the vectors $\mathbf{R}_{1}$ and $\mathbf{c}$ and a sharp decrease in the anisotropy energy.
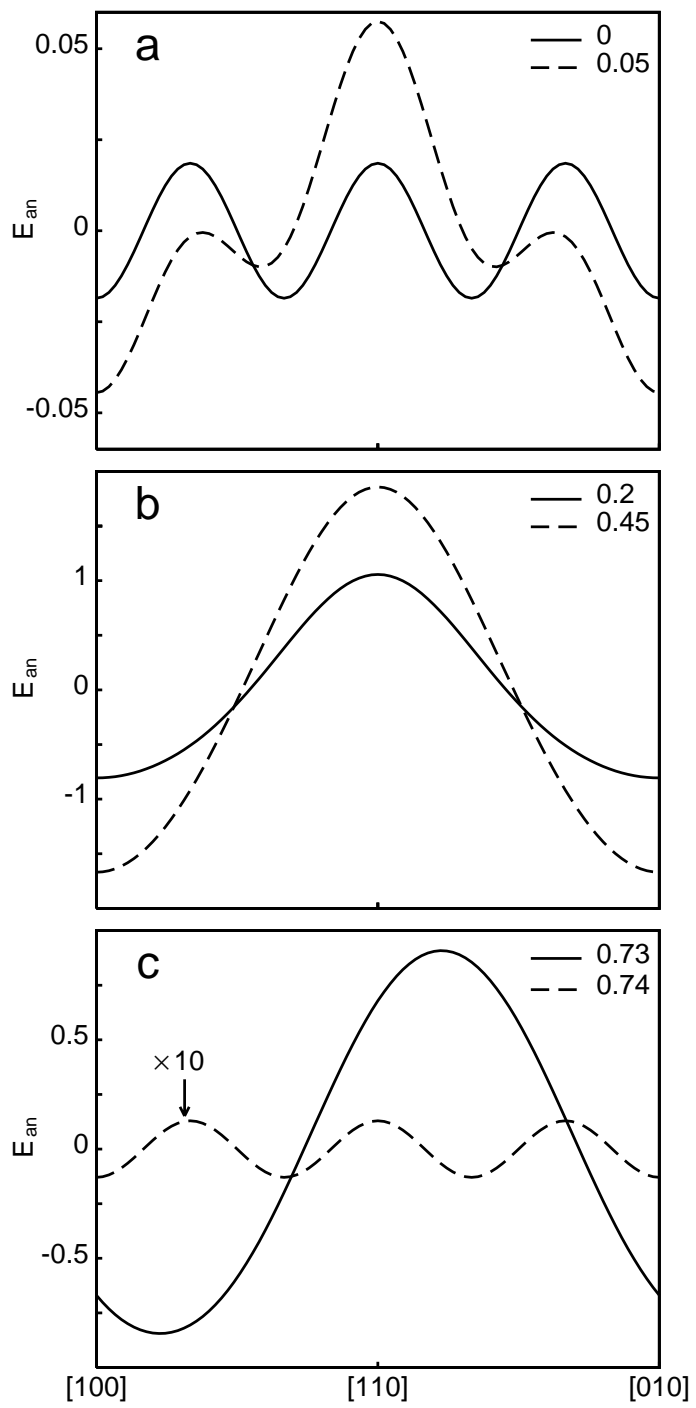

FIG. 2. The dependence of the anisotropic part of the piezoelectric correction to the energy of a double layer Wigner crystal in a (001) plane versus the direction of the $\mathbf{R}_{1}$ vector for the values of $\eta$ specified; a, a simple hexagonal phase and a rectangular phase at small $\eta$; b, a rectrangular phase at large $\eta$ and a square phase; $\mathrm{c}$, a rhombic and double hexagonal phases near the first-order transition point. The energy is given in units of $e^{2} \chi \sqrt{n} / 2 \varepsilon$. 
In the case of two-dimensional electron layers parallel to the (0-11) plane, the calculation gives the following results. A minimum of the energy of the simple hexagonal structure is reached when one of the primitive lattice vectors is oriented along the [100] axis. When the distance between layers is increased and the rectangular structure is formed, there arise two distinct equilibrium orientations, one of which corresponds to a local minimum $\left(\mathbf{R}_{1}\right.$ directed along the [100] axis) and the other to a global minimum $\left(\mathbf{R}_{1}\right.$ directed at an angle $\beta \approx 60^{\circ}$ to the [100] or [-100] axis). Near the points of transition to the square phase the local minimum vanishes, and a rapid reorientation of the electron lattice occurs. The energy of the square phase is minimum when one of the primitive lattice vectors is directed at an angle $\beta=45^{\circ}$ to the [100] axis, i.e., to the vector $\mathbf{c}$, which is parallel to the [100] or the [011] axis. After the transition to the rhombic phase the direction of the vector $\mathbf{c}$ parallel to the [011] axis corresponds to the global minimum, while the direction parallel to the [100] axis corresponds to a local minimum. As $\eta$ is increased, the local minimum splits into two, corresponding to a direction of the vector $\mathbf{c}$ at an angle $\pm \beta\left(\beta<30^{\circ}\right)$ to this axis. At the transition to the double hexagonal phase all three minima become equal (the orientation of $\mathbf{R}_{1}$ changes abruptly at this transition).
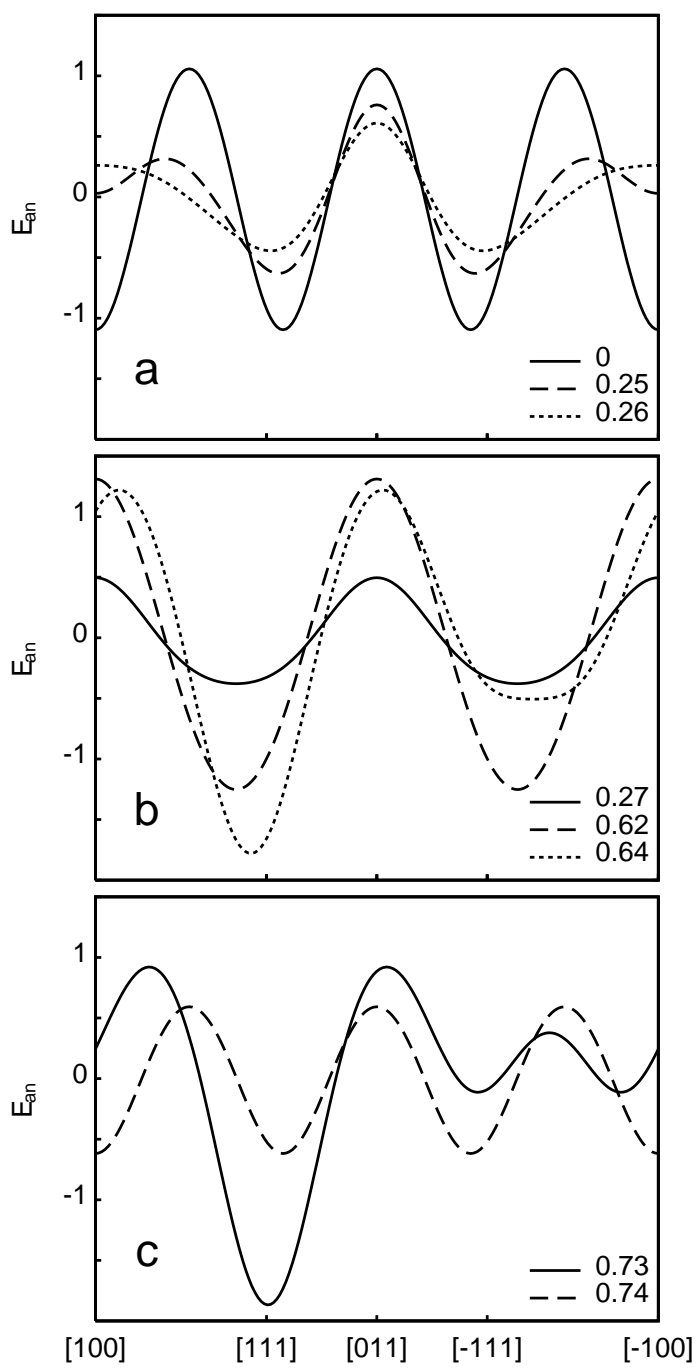

FIG. 3. The anisotropic part of the energy of the Wigner crystal in a (0-11) plane versus the direction of the $\mathbf{R}_{1}$ vector for the values of $\eta$ specified; a, a simple hexagonal and a rectangular phases; $\mathrm{b}$, a square and rhombic phases near the second-order transition point; c, a rhombic and double hexagonal phases near the first-order transition point. 
In a bilayer structure lying parallel to the (111) plane the minimum of the energy of the simple hexagonal and rectangular phases correspond to a direction of $\mathbf{R}_{1}$ at an angle $\beta=30^{\circ}$ to one of the twofold axes lying in the (111) plane. As the point of transition to the square phase is approached, the anisotropic contribution decreases sharply. A sharp reorientation occurs near the transition point. For the square phase a minimum of the energy is reached when the vector $\mathbf{R}_{1}$ is directed at an angle $\beta= \pm 15^{\circ}$ to one of the twofold axes. At the transition to the rhombic phase the anisotropy again increases. The energy of the rhombic phase is minimum in the case when the vector $\mathbf{c}$ is oriented along one of the twofold axes. At the transition to the double hexagonal phase the orientation of the vector c changes abruptly - it deviates somewhat from the twofold axis. We note that in the double hexagonal phase the energetically most favorable orientation of the lattice vectors differs from the case of the simple hexagonal phase. The latter is due to the absence of a center of inversion in the double hexagonal structure.
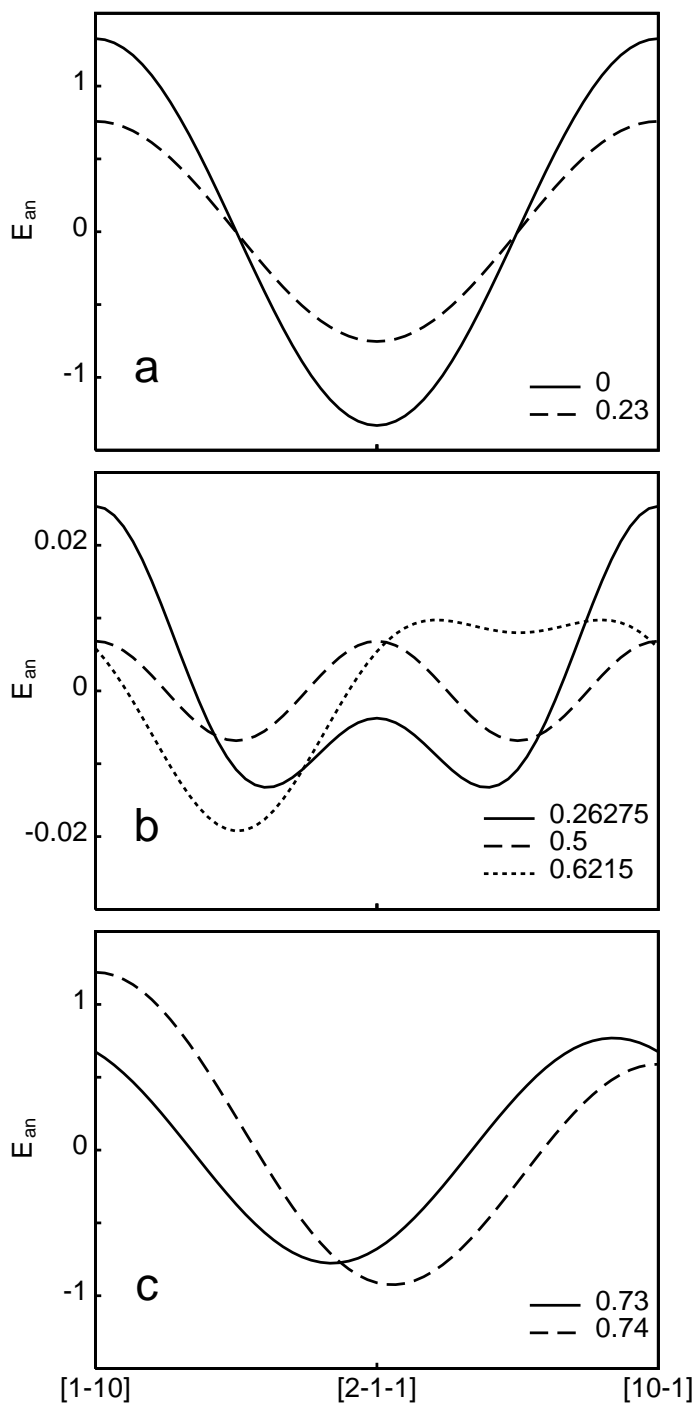

FIG. 4. The anisotropic part of the energy of the Wigner crystal in a (111) plane versus the direction of the $\mathbf{R}_{1}$ vector for the values of $\eta$ specified; a, a simple hexagonal and a rectangular phases; b, a rectangular and and a rhombic phases near the second-order transition point and a square phase; c, a rhombic and double hexagonal phases near the first-order transition point.

The results show that the orientation of a bilayer Wigner crystal in a piezoelectric matrix is determined by the plane in which the electron layer is located, the type of electron lattice, and the value of the parameter $\eta$. Interestingly, the 
abrupt (jumplike) character of the reorientation is observed not only at the transition from the rhombic to the double hexagonal phase (this is the expected effect, since it accompanies a first-order transition). A very rapid reorientation also takes place at the second-order transition from the rectangular to the square phase.

The value of the anisotropy energy is determined by the parameter $\chi$, which in GaAs is of the order of $2 \times 10^{-4}$. The typical difference between the Coulomb energy in the different phases is of the order of $10^{-2} e^{2} \sqrt{n} / \varepsilon$ per electron, [7] 8 ] i.e., according to the results reported, the piezoelectric interaction in the system under study is approximately two orders of magnitude smaller; hence, it has a weak influence on the phase diagram and only determines the orientation of the electron crystal. Nevertheless, in other systems in which the value of the piezoelectric modulus is larger, one can expect a radical rearrangement of the phase diagram. Analogous effects for a monolayer system were discussed in Ref. [4]. The approach considered in the present paper makes it possible to investigate this possibility in detail for the case of bilayer electron crystals.

\section{APPENDIX}

Let us transform to the rapidly convergent form of the expression

$$
S_{ \pm m}=\sum_{\mathbf{R} \neq 0} \frac{e^{ \pm i m \psi_{\mathbf{R}}}}{R}
$$

where $m>0$. We introduce the function

$$
T_{ \pm m}(\mathbf{r}, \mathbf{q})=e^{-i \mathbf{q r}} \sum_{|\mathbf{r}+\mathbf{R}|} \frac{e^{i \mathbf{q}(\mathbf{R}+\mathbf{r}) \pm i m \psi_{\mathbf{r}+\mathbf{R}}}}{|\mathbf{r}+\mathbf{R}|}-\frac{e^{ \pm i m \psi_{\mathbf{r}}}}{r}
$$

such that

$$
S_{ \pm m}=\lim _{\mathbf{r} \rightarrow 0, \mathbf{q} \rightarrow 0} T_{ \pm m}(\mathbf{r}, \mathbf{q})
$$

We use the identity

$$
\frac{\gamma\left(\frac{m+1}{2}, x\right)+\Gamma\left(\frac{m+1}{2}, x\right)}{\Gamma\left(\frac{m+1}{2}\right)} \equiv 1
$$

With allowance for (A.4) the quantity $T_{ \pm m}$ can be written in the form a sum

$$
T_{ \pm m}(\mathbf{r}, \mathbf{q})=T_{ \pm m, 1}(\mathbf{r}, \mathbf{q})+T_{ \pm m, 2}(\mathbf{r}, \mathbf{q})
$$

where

$$
\begin{gathered}
T_{ \pm m, 1}(\mathbf{r}, \mathbf{q})=\sum_{\mathbf{R} \neq 0} \frac{e^{i \mathbf{q} \mathbf{R} \pm i m \psi_{\mathbf{r}+\mathbf{R}}}}{|\mathbf{r}+\mathbf{R}|} \frac{\Gamma\left(\frac{m+1}{2}, \pi n|\mathbf{r}+\mathbf{R}|^{2}\right)}{\Gamma\left(\frac{m+1}{2}\right)}-\frac{e^{ \pm i m \psi_{\mathbf{r}}}}{r} \frac{\gamma\left(\frac{m+1}{2}, \pi n r^{2}\right)}{\Gamma\left(\frac{m+1}{2}\right)} \\
T_{ \pm m, 2}(\mathbf{r}, \mathbf{q})=e^{-i \mathbf{q r}} \sum_{\mathbf{R}} \frac{e^{i \mathbf{q}(\mathbf{r}+\mathbf{R}) \pm i m \psi_{\mathbf{r}+\mathbf{R}}}}{|\mathbf{r}+\mathbf{R}|} \frac{\gamma\left(\frac{m+1}{2}, \pi n|\mathbf{r}+\mathbf{R}|^{2}\right)}{\Gamma\left(\frac{m+1}{2}\right)}
\end{gathered}
$$

We note that the last term in (A.6) vanishes in the limit $\mathbf{r} \rightarrow 0$.

For the transformation $T_{ \pm m, 2}$ we substitute the definition of the function $\gamma(\alpha, x)$ into (A.7):

$$
T_{ \pm m, 2}(\mathbf{r}, \mathbf{q})=\frac{2}{\Gamma\left(\frac{m+1}{2}\right)} e^{-i \mathbf{q r}} \int_{0}^{\sqrt{\pi n}} d \xi \xi^{m} \sum_{\mathbf{R}}|\mathbf{r}+\mathbf{R}|^{m} e^{i \mathbf{q}(\mathbf{r}+\mathbf{R}) \pm i m \psi_{\mathbf{r}+\mathbf{R}}-\xi^{2}|\mathbf{r}+\mathbf{R}|^{2}}
$$

Expanding (A.8) in a Fourier series in the reciprocal lattice vectors, we get

$$
T_{ \pm m, 2}(\mathbf{r}, \mathbf{q})=\frac{2 n}{\Gamma\left(\frac{m+1}{2}\right)} \sum_{\mathbf{G}} e^{-i(\mathbf{q}+\mathbf{G}) \mathbf{r}} \int_{0}^{\sqrt{\pi n}} d \xi \xi^{m} \int d^{2} \boldsymbol{\rho} \rho^{m} \exp \left[i \boldsymbol{\rho}(\mathbf{q}+\mathbf{G}) \pm i m \psi \boldsymbol{\rho}-\xi^{2} \rho^{2}\right]
$$


Evaluating the integral over $\boldsymbol{\rho}$, we find

$$
T_{ \pm m, 2}(\mathbf{r}, \mathbf{q})=i^{m} \frac{2 \pi n}{\Gamma\left(\frac{m+1}{2}\right)} \sum_{\mathbf{G}} e^{-i(\mathbf{q}+\mathbf{G}) \mathbf{r} \pm i m \psi_{\mathbf{q}+\mathbf{G}}}\left(\frac{|\mathbf{q}+\mathbf{G}|}{2}\right)^{m} \int_{0}^{\sqrt{\pi n}} d \xi \frac{1}{\xi^{m+2}} \exp \left(-\frac{|\mathbf{q}+\mathbf{G}|^{2}}{4 \xi^{2}}\right) .
$$

Making the change of variables $\xi=|\mathbf{q}+\mathbf{G}| / 2 t$, we arrive at the form

$$
T_{ \pm m, 2}(\mathbf{r}, \mathbf{q})=i^{m} \frac{2 \pi n}{\Gamma\left(\frac{m+1}{2}\right)} \sum_{\mathbf{G}} e^{-i(\mathbf{q}+\mathbf{G}) \mathbf{r} \pm i m \psi_{\mathbf{q}+\mathbf{G}}} \frac{1}{|\mathbf{q}+\mathbf{G}|} \Gamma\left(\frac{m+1}{2}, \frac{|\mathbf{q}+\mathbf{G}|^{2}}{4 \pi n}\right) .
$$

Substituting formulas (A.1), (A.3), (A.7), and (A.11) into Eq. (12) and introducing the function (17), we arrive at Eq. (15). The term with $\mathbf{G}=0$ in (A.11) drops out, since it cancels with the intralayer interaction with the positive neutralizing background that appears in $E_{\mathrm{an}}^{B G}$.

An analogous transformation may be done for the sum

$$
S_{l, \pm m}=\sum_{\mathbf{R}} \frac{d^{l}|\mathbf{R}+\mathbf{c}|^{m} e^{ \pm i m \psi_{\mathbf{R}+\mathbf{c}}}}{\left(|\mathbf{R}+\mathbf{c}|^{2}+d^{2}\right)^{(l+m+1) / 2}} .
$$

Here we also use the identity (A.4), with $m$ replaced by $m+l$. We get

$$
S_{l, \pm m}=T_{l, \pm m, 1}(0,0)+\lim _{\mathbf{r} \rightarrow 0, \mathbf{q} \rightarrow 0} T_{l, \pm m, 2}(\mathbf{r}, \mathbf{q}),
$$

where

$$
T_{l, \pm m, 1}(0,0)=\sum_{\mathbf{R}} \frac{d^{l}|\mathbf{R}+\mathbf{c}|^{m} e^{ \pm i m \psi_{\mathbf{R}+\mathbf{c}}}}{\left(|\mathbf{R}+\mathbf{c}|^{2}+d^{2}\right)^{(l+m+1) / 2}} \frac{\Gamma\left[\frac{l+m+1}{2}, \pi n\left(|\mathbf{R}+\mathbf{c}|^{2}+d^{2}\right)\right]}{\Gamma\left(\frac{l+m+1}{2}\right)} .
$$
form

The quantity $T_{l, \pm m, 2}(\mathbf{r}, \mathbf{q})$, after we change to summation over $\mathbf{G}$ and do the integration over $\boldsymbol{\rho}$, reduces to the

$$
\begin{aligned}
T_{l, \pm m, 2}(\mathbf{r}, \mathbf{q})=i^{m} \frac{2 \pi n}{\Gamma\left(\frac{l+m+1}{2}\right)} & \sum_{\mathbf{G}} e^{-i \mathbf{G} \mathbf{c}-i(\mathbf{q}+\mathbf{G}) \mathbf{r} \pm i m \psi_{\mathbf{q}+\mathbf{G}}} d^{l}\left(\frac{|\mathbf{q}+\mathbf{G}|}{2}\right)^{m} \\
\times & \int_{0}^{\sqrt{\pi n}} d \xi \xi^{l-m-2} \exp \left(-\xi^{2} d^{2}-\frac{|\mathbf{q}+\mathbf{G}|^{2}}{4 \xi^{2}}\right) .
\end{aligned}
$$

Evaluation of the integral in (A.15) gives

$$
\begin{aligned}
T_{l, \pm m, 2}(\mathbf{r}, \mathbf{q})=i^{m} & \frac{\pi n}{\Gamma\left(\frac{l+m+1}{2}\right)} \sum_{\mathbf{G}} e^{-i \mathbf{G c}-i(\mathbf{q}+\mathbf{G}) \mathbf{r} \pm i m \psi_{\mathbf{q}+\mathbf{G}}} \frac{1}{|\mathbf{q}+\mathbf{G}|} \sum_{s=0}^{N(l, m)} C_{N(l, m)+s}^{2 s}\left(\frac{|\mathbf{q}+\mathbf{G}| d}{2}\right)^{\frac{m+l-2 s}{2}} \\
& \times\left\{e^{-|\mathbf{q}+\mathbf{G}| d}\left[\Gamma\left(s+\frac{1}{2}\right)-\operatorname{sgn}\left(f_{-}\right) \gamma\left(s+\frac{1}{2}, f_{-}^{2}\right)\right]+(-1)^{\frac{l+m-2 s}{2}} e^{|\mathbf{q}+\mathbf{G}| d} \Gamma\left(s+\frac{1}{2}, f_{+}^{2}\right)\right\},
\end{aligned}
$$

where

$$
\begin{gathered}
f_{ \pm}=\frac{|\mathbf{q}+\mathbf{G}|}{2 \sqrt{\pi n}} \pm \sqrt{\pi n} d \\
N(l, m)=\max \left(\frac{m-l}{2}, \frac{l-m-2}{2}\right) .
\end{gathered}
$$

In deriving (A.16) we took into account that in the problem considered, the parameters $l$ and $m$ have the same parity. The term with $\mathbf{G}=0$ in (A.16) is compensated by the interaction with the positive background of the adjacent layer. Using (A.13), (A.14), and (A.16), we can write the contribution of the interlayer interaction in the form (16). 
[1] L. Brey, H. A. Fertig, R. Cote, and A. H. McDonald, Phys. Rev. Lett. 75, 2562 (1995).

[2] A. A. Koulakov, M. M. Fogler, and B. I. Shklovskii, Phys. Rev. Lett. 76, 499 (1996); M. M. Fogler, A. A. Koulakov, and B. I. Shklovskii, Phys. Rev. B 54, 1853 (1996).

[3] M. P. Lilly, K. B. Cooper, J. P. Eisenstein, L. N. Pfeiffer, and K. W. West, Phys. Rev. Lett. 82, 394 (1999); R. R. Du, D. C. Tsui, H. L. Stormer, L. N. Pfeiffer, and K. W. West, Solid State Commun. 109, 389 (1999).

[4] É. I. Rashba and E. Ya. Sherman, Fiz. Tekh. Poluprovodn. 21, 1957 (1987) [Sov. J. Semicond. 21, 1185 (1987)].

[5] E. Ya. Sherman, Phys. Rev. B 52, 1512 (1995).

[6] D. V. Fil, Low Temp. Phys. 26, 581 (2000) [Fiz. Nizk. Temp. 26, 792 (2000)]; D. V. Fil, cond-mat/0004107.

[7] L. Bonsall and A. A. Maradudin, Phys. Rev. B 15, 1959 (1977).

[8] G. Goldoni and F. M. Peeters, Phys. Rev. B 53, 4591 (1996).

[9] I. V. Schweigert, V. A. Schweigert, and F. M. Peeters, Phys. Rev. Lett. 82, 5293 (1999).

[10] I. V. Schweigert, V. A. Schweigert, F. M. Peeters, Phys. Rev. B 60, 14665 (1999).

[11] L. Zheng, H. A. Fertig, Phys. Rev. B 52, 12282 (1995).

[12] S. Narasimhan, T. L. Ho, Phys. Rev. B 52, 12291 (1995).

[13] Y. M. Vil'k, Y. P. Monarkha, Sov. J. Low Temp. Phys. 10, 465 (1984) [Fiz. Nizk. Temp. 10, 886 (1984)].

[14] Y. M. Vil'k, Y. P. Monarkha, Sov. J. Low Temp. Phys. 11, 535 (1985) [Fiz. Nizk. Temp. 11, 971 (1985)]. 